\title{
Methicillin-resistant Staphylococcus aureus (MRSA) catheter-related bacteraemia in haemodialysis patients
}

Guillermo Cuervo ${ }^{*}$, Mariana Camoez ${ }^{2}$,Evelyn Shaw ${ }^{1}$, María Ángeles Dominguez², Oriol Gasch³ ${ }^{3}$ Belén Padilla ${ }^{4}$, Vicente Pintado ${ }^{5}$, Benito Almirante ${ }^{6}$, José Molina ${ }^{7}$, Francisco López-Medrano ${ }^{8}$, Enrique Ruiz de Gopegui ${ }^{9}$, José A. Martinez ${ }^{10}$, Elena Bereciartua ${ }^{11}$, Fernando Rodriguez-Lopez ${ }^{12}$, Carlos Fernandez-Mazarrasa ${ }^{13}$, Miguel Ángel Goenaga ${ }^{14}$, Natividad Benito ${ }^{15}$, Jesús Rodriguez-Baño ${ }^{16}$, Elena Espejo ${ }^{17}$, Miquel Pujol ${ }^{1}$ and On behalf of the REIPI/GEIH study group

\begin{abstract}
Background: The aim of the study was to determine clinical and microbiological differences between patients with methicillin-resistant Staphylococcus aureus (MRSA) catheter-related bacteraemia (CRB) undergoing or not undergoing haemodialysis, and to compare outcomes.

Methods: Prospective multicentre study conducted at 21 Spanish hospitals of patients with MRSA bacteraemia diagnosed between June 2008 and December 2009. Patients with MRSA-CRB were selected. Data of patients on haemodialysis (HD-CRB) and those not on haemodialysis (non-HD-CRB) were compared.
\end{abstract}

Results: Among 579 episodes of MRSA bacteraemia, 218 (37.7\%) were CRB. Thirty-four (15.6\%) were HD-CRB and 184 (84.4\%) non-HD-CRB. All HD-CRB patients acquired the infection at dialysis centres, while in $85.3 \%$ of the non-HD-CRB group the infection was nosocomial $(p<.001)$. There were no differences in age, gender or severity of bacteraemia (Pitt score); comorbidities (Charlson score $\geq 4$ ) were higher in the HD-CRB group than in the non-HD-CRB group (73.5 \% vs. $46.2 \%, p=.003)$. Although there were no differences in VAN-MIC $\geq 1.5 \mathrm{mg} / \mathrm{L}$ according to microdilution, using the E-test a higher rate of VAN-MIC $\geq 1.5 \mathrm{mg} / \mathrm{L}$ was observed in HD-CRB than in non-HD-CRB patients (63.3\% vs. $44.1 \%, p=.051)$. Vancomycin was more frequently administered in the HD-CRB group than in the non-HD-CRB group ( $82.3 \%$ vs. $42.4 \%, p=<.001$ ) and therefore the appropriate empirical therapy was significantly higher in HD-CRB group ( $91.2 \%$ vs. $73.9 \%, p=.029$ ). There were no differences with regard to catheter removal (79.4\% vs. $84.2 \%, p=.555$, respectively). No significant differences in mortality rate were observed between both groups (Overall mortality: $11.8 \%$ vs. $27.2 \%, p=.081$, respectively), but there was a trend towards a higher recurrence rate in HD-CRB group ( $8.8 \%$ vs. $2.2 \%, p=.076$ ).

Conclusions: In our multicentre study, ambulatory patients in chronic haemodialysis represented a significant proportion of cases of MRSA catheter-related bacteraemia. Although haemodialysis patients with MRSA catheter-related bacteraemia had significantly more comorbidities and higher proportion of strains with reduced vancomycin susceptibility than non-haemodialysis patients, overall mortality between both groups was similar.

Keywords: Bacteraemia, Catheter-related, Haemodialysis, MRSA

\footnotetext{
* Correspondence: guillermocuervo@hotmail.com

'Department of Infectious Diseases, Hospital Universitari de Bellvitge; Feixa

Llarga s/n, 08907, L'Hospitalet de Llobregat, Barcelona, Spain

Full list of author information is available at the end of the article
} 


\section{Background}

Methicillin-resistant Staphylococcus aureus (MRSA) bloodstream infection (BSI) has been a cause of major concern in healthcare systems all over the world, due to its high incidence rates and undesirable related outcomes [1-3]. Most series have found that the vascular catheter is the most frequent source of MRSA-BSI $[2,3]$.

Chronic haemodialysis patients, especially those dialysed through a venous catheter [4-6] are at a particularly high risk of this infection, with a rate more than 100 times higher than non-dialysis patients $[4,7]$. Among haemodialysis patients with staphylococcal infections, those with MRSA infections have significantly longer hospitalizations, with higher costs, and are more likely to die within three months than those with methicillin-susceptible Staphylococcus aureus (MSSA) infections [8].

Patients with MRSA infections often receive antibiotic treatment with glycopeptides [9]. Some reports have raised concerns about the observation of higher vancomycin MICs in isolates of patients with MRSA bacteraemia who had received vancomycin within the last 30 days $[10,11]$, and other authors have also documented a significant risk for treatment failure and a higher mortality with increasing vancomycin MIC, even if MICs are in the susceptible range [12, 13]. Similar findings were reported specifically in patients undergoing haemodialysis, in whom a higher mortality and a longer mean hospital length of stay were also observed, with an ensuing increase in hospital costs [14].

Current information on this topic is still scarce in Europe, particularly in patients receiving haemodialysis who develop catheter-related MRSA bacteraemia (HD-CRB). We aimed to analyse this issue in a large multicentre prospective cohort of patients with MRSA bacteraemia from Spain. Our objective was to compare the clinical presentation, microbiological characteristics and outcomes in this group of patients and in patients with MRSA catheter-related bacteraemia who were not receiving HD (non-HD-CRB).

\section{Methods}

\section{Study period and patients}

This was post-hoc analysis of a prospective multicentre study of all consecutive episodes of MRSA bacteraemia in hospitalized adult patients occurring from June 2008 to December 2009 at 21 Spanish hospitals. The following information was collected from medical records using a standardized protocol: demographic characteristics, comorbidities, clinical features, antibiotic therapy, and outcomes. Patients with catheter-related bacteraemia (CRB) were selected for the study, and those undergoing haemodialysis (HD-CRB) were compared with those who were not (non-HD-CRB).

\section{Definitions}

MRSA bacteraemia was defined as the presence of at least one positive blood culture for MRSA in a patient with clinical signs and symptoms of sepsis [15]. Vascular catheter-related bacteraemia was diagnosed using clinical and microbiological criteria defined by the guidelines of the Infectious Diseases Society of America [16]. It was considered when MRSA grew from at least one percutaneous blood sample culture and from a culture of the catheter tip, or when it grew in two blood samples for culture (one from a catheter hub and the other from a peripheral vein) that met criteria for quantitative blood cultures or differential time to positivity in a patient with accompanying clinical signs of sepsis and no other apparent source of infection. Complicated bacteraemia was defined as those episodes with positive follow-up blood cultures performed at 2-4 days after the beginning of adequate therapy and/ or with evidence of metastatic infection or endocarditis [17]. Comorbidity was measured by the Charlson score, as described elsewhere [18]. Patients were classified into three categories on the McCabe and Jackson scale [19] according to their prognosis of survival before the MRSA bacteraemia: rapidly fatal (death expected within the following year), ultimately fatal (death expected within a period of 1 to 5 years), and non fatal (life expectancy of $>5$ years). Severity of sepsis in the acute condition was assessed by the Pitt score [20]. Three acquisition categories were considered according to the Friedman criteria [21]: nosocomial, healthcare-related, and community-acquired bacteraemia. However, since all cases had healthcare exposure (i.e., dialysis) prior to culture, no community-associated cases were included. Distant extension was diagnosed in the presence of at least one distant infection secondary to blood spread seeding. The empirical antibiotic was defined as the antibiotic administered in the first $48 \mathrm{~h}$ after a positive blood culture was drawn, and it was considered appropriate if the strain was susceptible to at least one of the antibiotics administered according to the current CLSI breakpoints [22]. All patients were followed up to 4 weeks after completion of antibiotic treatment whether they were hospitalized or not. Recurrence within this period of time was defined as the isolation of MRSA in blood cultures after documented negative blood cultures or a newly diagnosed metastatic focus of the bacteremic past infection. Overall mortality was defined as death from any cause occurring in the 30 days and early mortality (EM) was defined for patients who died within the first two days after the onset of MRSA bacteraemia.

\section{Susceptibility testing and molecular epidemiology of MRSA isolates}

MRSA strains were identified in each hospital, where preliminary susceptibility tests were performed. Isolates 
were then sent to a central reference laboratory. All $S$. aureus were identified by latex agglutination (Pastorex Staph-plus, Bio-Rad Laboratories, Madrid, Spain) and DNase production (DNasE-test Agar, BioMérieux, Marcy l'Etoile, France). Antimicrobial susceptibility was tested by the disc diffusion method according to the CLSI guidelines [22]. MICs were determined by the microdilution method in accordance with CLSI criteria by using commercial panels (ESTEN 2009, SensititreTM, Izasa, Barcelona, Spain) read visually. Vancomycin E-test (BioMérieux) MICs were determined using a 0.5 McFarland inoculum streaked evenly with a swab onto Mueller-Hinton agar plates [23]. PFGE was performed after SmaI restriction of chromosomal DNA [24]. Restriction patterns were interpreted in accordance with criteria published elsewhere [25]. Representative isolates of each PFGE type and subtype were studied to determine the Multilocus Sequence Type (MLST) [26] and the Staphylococcal Chromosome Cassette mec (SCCmec) types [27]. MLSTs and SCCmec types were further inferred for all the strains. The agr polymorphism and the presence of genes encoding class $\mathrm{S}$ (lukS-PV) and class $\mathrm{F}$ (lukF-PV) proteins for Panton-Valentine Leukocidin (PVL) were studied by PCR in all the isolates, following the methodology described elsewhere [28, 29].

\section{Statistical analysis}

Continuous variables were compared using the Student's $t$-test or the Mann-Whitney $U$-test as appropriate. Categorical data were compared using Fisher's exact or Chi-squared tests. Analyses were performed using SPSS v15 (SPSS Inc., Chicago, IL, USA).

\section{Ethical considerations}

This observational study was conducted in accordance with the Declaration of Helsinki and was approved by the Spanish Network for Research in Infectious Diseases (REIPI) and by the Institutional Review Board at each participating centre: H. Arnau de Vilanova,
Lleida; H. Universitari de Bellvitge, Barcelona; H. de Burgos, Burgos; H. Clìnic, Barcelona; H. Universitario 12 de Octubre, Madrid; H. de Cruces, Barakaldo; H. de Donostia, Donostia; H. General Universitario Gregorio Marañón, Madrid; H. Universitari Joan XXIII, Tarragona; H. Universitari del Mar, Barcelona; H. Universitario Marques de Valdecilla, Santander; H. Universitari Mutua de Terrassa, Terrassa; H. del Parc Taulí, Sabadell; H. Universitario Ramon y Cajal, Madrid; H. Universitario Reina Sofía, Córdoba; H. San Pedro de la Rioja, Logroño; H. de la Santa Creu i Sant Pau, Barcelona; H. Universitari Son Espases, Palma de Mallorca; H. Universitario Virgen Macarena, Sevilla; H. Universitario Virgen del Rocío, Sevilla; H. Universitari Vall d'Hebrón, Barcelona. To protect personal privacy, identifying information of each patient in the electronic database was encrypted. Informed consent was waived by the Clinical Research Ethics Committee because no intervention was involved and no information able to identify the patient was included.

\section{Results}

\section{Characteristics of patients}

From a total of 579 episodes of MRSA bacteraemia, 218 (37.7 \%) were catheter-related (Fig. 1): 34 (15.6 \%) occurred in the HD-CRB group and 184 (84.4\%) in the non-HD-CRB group. The clinical and microbiological characteristics of patients are shown in Table 1. The comparison between the groups did not find significant differences in gender or age distribution. All HD-CRB patients acquired the infection at dialysis centres (chronic ambulatory haemodialysis), while the acquisition in non-HD-CRB patients was nosocomial in $85.3 \%$ of cases $(p<.001)$. Comorbidities measured by the Charlson score were higher in the HD-CRB group (Charlson $\geq 4: n=25,73.5 \%$ vs. $n=85,46.2 \%, p=.003$ ) while the severity of the underlying disease according to the McCabe scale was lower in this group (McCabe $\geq 2$ : $n=13,38.2 \%$ vs. $n=97,52.7 \%, p=.120$ ). No differences

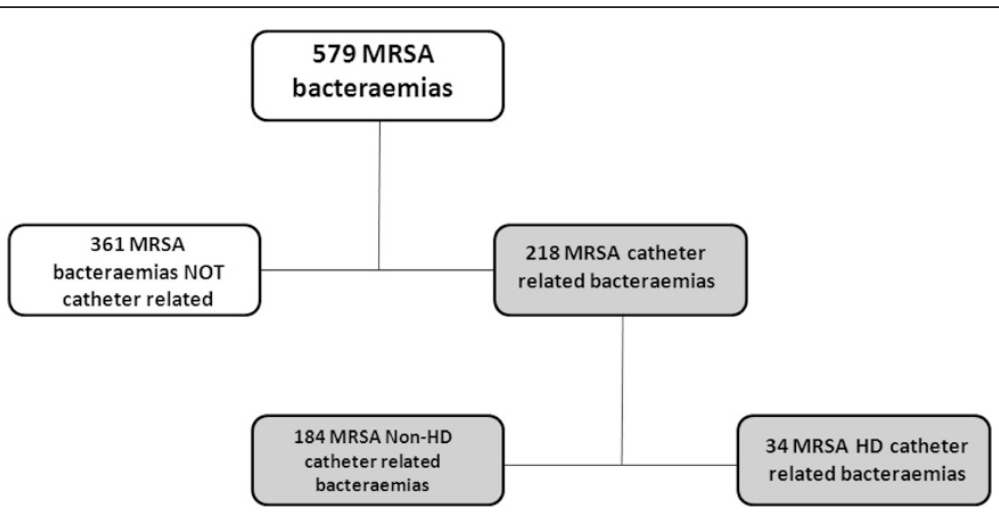

Fig. 1 Flow chart of patients included in analysis 
Table 1 Clinical and microbiological characteristics of patients by group

\begin{tabular}{|c|c|c|c|}
\hline & $\begin{array}{l}\text { HD-CRB } \\
n=34(100 \%)\end{array}$ & $\begin{array}{l}\text { Non-HD-CRB } \\
n=184(100 \%)\end{array}$ & $p$ \\
\hline Male Sex & $23(67.6)$ & $113(61.4)$ & .491 \\
\hline Age $>70$ y & $16(47.1)$ & $92(50)$ & .753 \\
\hline \multicolumn{4}{|l|}{ Acquisition } \\
\hline Health-care & $34(100)$ & $27(14.7)$ & $<.001$ \\
\hline Nosocomial & 0 & $157(85.3)$ & $<.001$ \\
\hline Charlson score $>4$ & $25(73.5)$ & $85(46.2)$ & .003 \\
\hline McCabe scale > 2 & $13(38.2)$ & $97(52.7)$ & .120 \\
\hline Pitt score > 3 & $6(17.6)$ & $55(29.9)$ & .144 \\
\hline \multicolumn{4}{|l|}{ Microbiology } \\
\hline agr type ${ }^{a}$ & $3(10)$ & $44(24.9)$ & .082 \\
\hline$\|$ & $27(90)$ & $132(74.6)$ & .354 \\
\hline PFGE type 2 & $25(83.3)$ & $107(60.5)$ & .091 \\
\hline 4 & $2(6.7)$ & $18(10.2)$ & .688 \\
\hline Other & $3(10)$ & $52(29.3)$ & .046 \\
\hline \multicolumn{4}{|l|}{ Clonal Complex ${ }^{\text {b }}$} \\
\hline 5 & $28(93.3)$ & $128(74.4)$ & .128 \\
\hline 8 & 0 & $14(8.1)$ & .199 \\
\hline 22 & $2(6.7)$ & $17(9.9)$ & .759 \\
\hline Other & 0 & $13(7.6)$ & .774 \\
\hline$P V L^{a}$ & 0 & $2(1.1)$ & 1 \\
\hline $\begin{array}{l}\text { Microdilution Vancomycin } \\
\mathrm{MIC}^{\mathrm{a}}>=1.5 \mathrm{mg} / \mathrm{L}\end{array}$ & 0 & $(4.9)$ & .363 \\
\hline $\begin{array}{l}\text { E-test Vancomycin } \\
\mathrm{MIC}^{\mathrm{a}}>=1.5 \mathrm{mg} / \mathrm{L}\end{array}$ & $19(63.3)$ & $78(44.1)$ & .051 \\
\hline
\end{tabular}

Data available in 207 patients

${ }^{b}$ Data available in 202 patients. Within Clonal Complex 5, 124 isolates belonged to the ST125 clone (HD-CRB: $25 / 83.3 \%$ vs. Non-HD-CRB: $99 / 57.6 \%$; $p=.013$ ) whereas 9 isolates belonged to the ST146 clone (HD-CRB: $2 / 6.7 \%$ vs. Non-HD-CRB: $7 / 4 \% ; p=.875$ )

Abbreviations: $H D$-CRB Haemodialysis catheter-related bacteraemia, Non-HD$C R B$ Non-haemodialysis catheter-related bacteraemia, agr Accessory gene regulator, PFGE Pulsed field gel electrophoresis, PVL Panton Valentine Leukocidin, MIC Minimum inhibitory concentration

were observed between groups in the severity of bacteraemia measured by the Pitt score.

\section{Microbiologic studies}

A dominant PFGE genotype (pulse-type 2) was found in both groups (Table 1), with all isolates belonging to Clonal Complex (CC) 5 (ST125 and ST146). They carried a SCCmec element type IV and agr type 2. PVL was positive in only two isolates in the non-HDCRB group, both belonging to the USA300 clone. No isolates had vancomycin $\mathrm{MIC} \geq 1.5 \mathrm{mg} / \mathrm{L}$ tested with the broth microdilution method in the HD-CRB group, compared with seven isolates (5\%) in the non-HD-CRB group $(p=.60)$. However, when MIC was assessed by the E-test, 19 patients $(63.3 \%)$ in the
HD-CRB group had vancomycin $\mathrm{MIC} \geq 1.5 \mathrm{mg} / \mathrm{L}$, and $78(44.1 \%)$ in the non-HD-CRB $(p=.051)$.

\section{Treatment and outcomes}

The treatment and clinical outcomes are detailed in Table 2. Empirical therapy was considered appropriate in 31 patients $(91.2 \%)$ in the HD-CRB group vs. $118(64.1 \%)$ in the non-HD-CRB group $(p=.001)$. Vancomycin was the most frequent empirical therapy in the HD-CRB group, in which 28 patients received vancomycin treatment $(82.4 \%)$ versus 78 patients $(42.4 \%)$ in the non-HD-CRB group $(p=<.001)$. There were no significant differences between groups in the number of catheters removed during the episode ( $n=27,79.4 \%$ vs. $n=155,84.2 \%, p=.555)$. Although no significant differences in outcomes were observed, there was a trend towards recurrent bacteraemia in the HD-CRB group $(3 / 34,8.8 \%$ vs. $n=4$ / $184,2.2 \%, p=.076)$.

\section{Discussion}

In this multicentre study of a large Spanish cohort including a high number of patients with MRSA catheterrelated bacteraemia, patients receiving haemodialysis did not present worse outcomes than the other group of patients with MRSA catheter-related bacteraemia. These findings challenge the results of previous reports which suggested that patients receiving HD are more prone to complications and poor prognosis [30, 31].

It is known that HD patients are frequently and recurrently colonized by MRSA [32, 33]. In fact, this pathological condition is a well known risk factor for MRSA bacteraemia [4, 34]. This is a matter of concern, as the size of the dialysis population is increasing worldwide

Table 2 Treatments and clinical outcomes of patients by group

\begin{tabular}{llll}
\hline & HD-CRB & Non-HD-CRB & $p$ \\
& $n=34(100 \%)$ & $n=184(100 \%)$ & \\
\hline Treatment (within $48 \mathrm{~h})$ & & & \\
Catheter removal & $27(79.4)$ & $155(84.2)$ & .555 \\
Vancomycin therapy & $28(82.3)$ & $78(42.4)$ & $<.001$ \\
Appropriate ATB therapy $^{\mathrm{a}}$ & $31(91.2)$ & $118(64.1)$ & .002 \\
Outcome & & & \\
Persistent bacteraemia 3d & $5(14.7)$ & $37(20.1)$ & .463 \\
Persistent bacteraemia 7d & $2(5.9)$ & $26(14.1)$ & .533 \\
Complicated bacteraemia & $11(32.4)$ & $89(48.4)$ & .095 \\
Distant secondary focus & $5(15.2)$ & $38(20.7)$ & .465 \\
Endocarditis & 0 & $10(5.4)$ & .368 \\
Recurrence & $3(8.8)$ & $4(2.2)$ & .076 \\
Early mortality (48 h) & 0 & $7(3.8)$ & .599 \\
Overall mortality (30d) & $4(11.8)$ & $49(27.2)$ & .081 \\
\hline
\end{tabular}

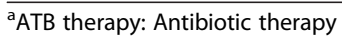


[35], as well as the proportion of HD patients using a catheter [36].

In our cohort of 579 MRSA patients, 218 (37.7\%) had catheter-related bacteraemia, a rate similar to other reports [37]. Patients on HD through a catheter had a greater burden of comorbidities as measured by Charlson score, a frequent finding in these patients [38].

Regarding vancomycin susceptibility tests, our findings reproduce the highly variable and method-dependent results already reported by some authors $[39,40]$. None of our HD-CRB patients had high MICs according to microdilution, but the E-test method identified high MICs in 19 of them. In spite of these E-test results, however, our HD patients did not have poorer outcomes, in agreement with other reports [14, 41]. In other words, the MIC measured by E-test did not improve the predictive ability of the microdilution in our cohort, which challenges us about its accuracy as a prognostic reference method. Vancomycin was the most frequently prescribed empirical therapy in our HD patients; its administration certainly is a common practice in this population [9]. The high presumption of MRSA in HD patients with a suspected infection may explain the choice of a more appropriate antibiotic therapy for this group both in our cohort and in others [42].

Although no significant differences in outcomes between groups were observed, HD patients had a tendency towards a higher recurrence rate, in agreement with previous research [43] but not a higher frequency of endocarditis [5]. Taken together, patients with MRSA catheter-related bacteraemia in our cohort had lower early and overall mortality rates $(3.2 \%$ and $24 \%$ respectively) compared with studies which analysed MRSA bacteraemia of all sources [44]. In fact, patients with catheter-related bacteraemia represent a particular group in which the main therapeutic strategy is catheter removal; this was achieved within $48 \mathrm{~h}$ in $80 \%$ of our patients. Furthermore, some authors have suggested that, rather than vancomycin MIC, the anatomical site of infection may be the best predictor of therapy success [42]. Finally, our HD patients exhibited a trend towards lower early and total mortality compared with the other group in which there was significantly more inappropriate initial treatment, a well known predictor of mortality [45]. In agreement with our findings, some recent reports on Staphylococcus aureus bacteraemia (both MSSA and MRSA) found lower 30 and 90 days case fatality rates in patients with end stage renal disease, irrespective of the type of replacement therapy received [46].

Our study has some limitations. First, it included a relatively low number of $\mathrm{HD}$ patients with $\mathrm{CRB}$, and its multicentre nature may have introduced some differences between centres regarding the clinical management. However, to our knowledge, it is the first study to address this specific population group within all patients with MRSA bacteraemia. On the other hand, isolates were frozen prior to MIC testing, which potentially could have underestimated MIC lecture in both groups [47].

\section{Conclusion}

In our multicentre study, ambulatory patients in chronic haemodialysis represented a significant proportion of cases of MRSA catheter-related bacteraemia. Although haemodialysis patients had significantly more comorbidities and higher proportion of strains with reduced vancomycin susceptibility than non-haemodialysis patients, they do not have worse outcomes. In fact, the higher frequency of appropriate empirical antimicrobial therapy may explain the trend towards better outcomes in this group, even though the sample size could prevent its statistical confirmation.

\section{Competing interests}

B. A. has received funding for research from Pfizer, Novartis, Gilead and MSD, and funds for advisory board membership from Pfizer, Gilead, Novartis, Janssen, Astellas and MSD. N. B. has received funding for speaking, consultancy, advisory board membership and travel from MSD, Pfizer, Gilead, Novartis and AstraZeneca. J. R.-B. has received funding for research from Novartis, has served as speaker for Astellas, Merck, Astra-Zeneca and Pfizer, and has been a consultant for Roche, Novartis and Janssen. All other authors: none to declare.

\section{Authors' contributions}

$M P, E S$ and GC conceived the study and participated in its design. GC wrote the manuscript. GC, MC,ES, MD, OG, BP, VP, BA, JM, FL-M, ER, JM, EB, FR-L, CF-M, $M G, N B, J R-B, E E$ and $M P$ critically revised the manuscript. All authors read and approved the final version for publication. REIPI/GEIH Study Groups were responsible for contributing patients in the study.

\section{Acknowledgements}

Writing assistance was provided by the Department of Languages in the Faculty of Medicine of Barcelona University.

\section{Members of the REIPI/GEIH Study Groups}

A Jover, F Barcenilla, M Garcia (H Arnau de Vilanova, Lleida, Spain); M Pujol, O Gasch, MaA Domínguez, M Camoez (H Universitari de Bellvitge, Barcelona, Spain); C Dueñas, E Ojeda (H de Burgos, Burgos Spain); JA Martinez, F bMarco (H Clìnic, Barcelona, Spain); F Chaves, M Lagarde, F López-Medrano (H Universitario 12 de Octubre, Madrid, Spain); JM Montejo, E Bereciartua, JL Hernández (H de Cruces, Barakaldo, Spain); MA Von Wichmann, MA Goenaga, JM García-Arenzana (H de Donostia, Donostia, Spain); B Padilla, C Padilla, E Cercenado (H General Universitario Gregorio Marañón, Madrid, Spain); G García-Pardo, J Tapiol (H Universitari Joan XXIII, Tarragona, Spain); JP Horcajada, M Montero, M Salvado (H Universitari del Mar, Barcelona, Spain); A Arnáiz, C Fernandez (H Universitario Marques de Valdecilla, Santander, Spain); E Calbo; M Xercavins (H Universitari Mutua de Terrassa, Terrassa, Spain); A Granados, D Fontanals (H del Parc Taulí, Sabadell, Spain); V Pintado, E Loza (H Universitario Ramon y Cajal, Madrid, Spain); J Torre-Cisneros, R Lara, F Rodríguez-López, M Rodríguez, C Natera (H Universitario Reina Sofía, Córdoba, Spain); JR Blanco, I Olarte (H San Pedro de la Rioja, Logroño, Spain); N Benito, B Mirelis (H de la Santa Creu i Sant Pau, Barcelona, Spain); J Murillas, E Ruiz de Gopegui (H Universitari Son Espases, Palma de Mallorca, Spain); E Espejo, MaA Morera (H de Terrassa, Terrassa, Spain); J Rodríguez-Baño, E López, A Pascual (H Universitario Virgen Macarena, Sevilla, Spain); C Martín, JA Lepe, J Molina (H Universitario Virgen del Rocío, Sevilla, Spain); R Sordé, B Almirante, N Larrosa (H Universitari Vall d'Hebrón, Barcelona, Spain). 


\section{Funding}

This study was supported by Ministerio de Ciencia e Innovación, Instituto de Salud Carlos III (FIS 08/0335) and co-financed by the European Development Regional Fund 'A way to achieve Europe' ERDF, Spanish Network for Research in Infectious Diseases (REIPI RD06/0008).

O. G. was recipient of a Río Hortega Grant (CM08/228) from the Instituto de Salud Carlos III.

\section{Author details}

'Department of Infectious Diseases, Hospital Universitari de Bellvitge; Feixa Llarga s/n, 08907, L'Hospitalet de Llobregat, Barcelona, Spain. ${ }^{2}$ Department of Microbiology, H. Bellvitge, Barcelona, Spain. ${ }^{3}$ Department of Infectious Diseases, H. Parc Taulí, Sabadell, Spain. ${ }^{4}$ Department of Infectious Diseases, H. Gregorio Marañón, Madrid, Spain. ${ }^{5}$ Department of Infectious Diseases, $H$. Ramón y Cajal, Madrid, Spain. ${ }^{6}$ Department of Infectious Diseases, H. Vall d'Hebrón, Barcelona, Spain. ${ }^{7}$ Department of Infectious Diseases, H. Virgen del Rocío, Sevilla, Spain. ${ }^{8}$ Department of Infectious Diseases, H. 12 de Octubre, Madrid, Spain. ${ }^{9}$ Department of Microbiology, H. Son Espases, Palma de Mallorca, Spain. ${ }^{10}$ Department of Infectious Diseases, H. Clinic, Barcelona, Spain. ${ }^{11}$ Department of Infectious Diseases, H. Cruces, Bilbao, Spain. ${ }^{12}$ Department of Infectious Diseases, H. Reina Sofía, Córdoba, Spain. ${ }^{13}$ Department of Microbiology, H. Marqués de Valdecilla, Santander, Spain. ${ }^{14}$ Department of Infectious Diseases, H. Donostia, Donostia, Spain. ${ }^{15}$ Department of Infectious Diseases, H. de la Santa Creu i Sant Pau, Barcelona, Spain. ${ }^{16}$ Department of Infectious Diseases, H. Virgen de Macarena, Sevilla, Spain. ${ }^{17}$ Department of Infectious Diseases, H. Terrassa, Terrassa, Spain.

Received: 8 January 2015 Accepted: 19 October 2015 Published online: 30 October 2015

\section{References}

1. Klevens RM, Morrison MA, Nadle J, Petit S, Gershman K, Ray S, et al. Invasive methicillin-resistant Staphylococcus aureus infections in the United States. JAMA. 2007;298:1763-71.

2. Gasch O, Ayats J, Angeles Dominguez M, Tubau F, Liñares J, Peña C, et al. Epidemiology of methicillin-resistant Staphylococcus aureus (MRSA) bloodstream infection: secular trends over 19 years at a university hospital. Med (Baltimore). 2011;90:319-27.

3. Cosgrove SE, Sakoulas G, Perencevich EN, Schwaber MJ, Karchmer AW, Carmeli Y. Comparison of mortality associated with methicillin-resistant and methicillin-susceptible Staphylococcus aureus bacteremia: a meta-analysis. Clin Infect Dis. 2003;36:53-9.

4. Nguyen DB, Lessa FC, Belflower R, Mu Y, Wise M, Nadle J, et al. Invasive Methicillin-Resistant Staphylococcus aureus Infections Among Patients on Chronic Dialysis in the United States, 2005-2011. Clin Infect Dis. 2013;57:1393-400.

5. Fitzgerald SF, O'Gorman J, Morris-Downes MM, Crowley RK, Donlon S, Bajwa $R$, et al. A 12-year review of Staphylococcus aureus bloodstream infections in haemodialysis patients: more work to be done. J Hosp Infect. 2011;79:218-21.

6. Fram D, Okuno MFP, Taminato M, Ponzio V, Manfredi SR, Grothe C, et al. Risk factors for bloodstream infection in patients at a Brazilian hemodialysis center: a case-control study. BMC Infect Dis. 2015;15:158.

7. Collins AJ, Foley RN, Chavers B, Gilbertson D, Herzog C, Ishani A, et al. US Renal Data System 2013 Annual Data Report. Am J Kidney Dis. 2014;63:A7.

8. Reed SD, Friedman JY, Engemann JJ, Griffiths RI, Anstrom KJ, Kaye KS, et al. Costs and outcomes among hemodialysis-dependent patients with methicillin-resistant or methicillin-susceptible Staphylococcus aureus bacteremia. Infect Control Hosp Epidemiol. 2005;26:175-83.

9. Chan KE, Warren HS, Thadhani RI, Steele DJR, Hymes JL, Maddux FW, et al. Prevalence and Outcomes of Antimicrobial Treatment for Staphylococcus aureus Bacteremia in Outpatients with ESRD. J Am Soc Nephrol. 2012;23:1551-9.

10. Moise PA, Smyth DS, El-Fawal N, Robinson DA, Holden PN, Forrest A, et al. Microbiological effects of prior vancomycin use in patients with methicillinresistant Staphylococcus aureus bacteraemia. J Antimicrob Chemother. 2008:61:85-90.

11. Lubin AS, Snydman DR, Ruthazer R, Bide P, Golan Y. Predicting high vancomycin minimum inhibitory concentration in methicillin-resistant Staphylococcus aureus bloodstream infections. Clin Infect Dis. 2011;52:997-1002
12. Sakoulas G, Moise-Broder PA, Schentag J, Forrest A, Moellering RC, Eliopoulos GM. Relationship of MIC and bactericidal activity to efficacy of vancomycin for treatment of methicillin-resistant Staphylococcus aureus bacteremia. J Clin Microbiol. 2004;42:2398-402.

13. Soriano A, Marco F, Martínez JA, Pisos E, Almela M, Dimova VP, et al. Influence of vancomycin minimum inhibitory concentration on the treatment of methicillin-resistant Staphylococcus aureus bacteremia. Clin Infect Dis. 2008;46:193-200.

14. Maclayton DO, Suda KJ, Coval KA, York CB, Garey KW. Case-control study of the relationship between MRSA bacteremia with a vancomycin MIC of 2 microg/mL and risk factors, costs, and outcomes in inpatients undergoing hemodialysis. Clin Ther. 2006;28:1208-16.

15. Levy MM, Fink MP, Marshall JC, Abraham E, Angus D, Cook D, et al. 2001 SCCM/ESICM/ACCP/ATS/SIS International Sepsis Definitions Conference. Crit Care Med. 2003;31:1250-6.

16. Mermel LA, Allon M, Bouza E, Craven DE, Flynn P, O'Grady NP, et al. Clinical practice guidelines for the diagnosis and management of intravascular catheter-related infection: 2009 Update by the Infectious Diseases Society of America. Clin Infect Dis. 2009;49:1-45.

17. Liu C, Bayer A, Cosgrove SE, Daum RS, Fridkin SK, Gorwitz RJ, et al. Clinical practice guidelines by the infectious diseases society of america for the treatment of methicillin-resistant Staphylococcus aureus infections in adults and children. Clin Infect Dis. 2011;52:e18-55.

18. Charlson ME, Pompei P, Ales KL, MacKenzie CR. A new method of classifying prognostic comorbidity in longitudinal studies: development and validation. J Chronic Dis. 1987;40:373-83.

19. MCCABE WR, JACKSON GG. Gram-Negative Bacteremia: II. Clinical, Laboratory, and Therapeutic Observations. Arch Intern Med. 1962;1 10:856-64.

20. Chow JW, Yu VL. Combination antibiotic therapy versus monotherapy for gram-negative bacteraemia: a commentary. Int J Antimicrob Agents. 1999;11:7-12.

21. Friedman ND, Kaye KS, Stout JE, McGarry SA, Trivette SL, Briggs JP, et al. Health care-associated bloodstream infections in adults: a reason to change the accepted definition of community-acquired infections. Ann Intern Med. 2002;137:791-7.

22. CLSI. Performance Standards for Antimicrobial Susceptibility Testing; Twenty-Second Informational Supplement. Clin Lab Stand Inst. 2013;32:1-184.

23. Walsh TR, Bolmström A, Qwärnström A, Ho P, Wootton M, Howe RA, et al. Evaluation of current methods for detection of staphylococci with reduced susceptibility to glycopeptides. J Clin Microbiol. 2001;39:2439-44.

24. Chung M, de Lencastre H, Matthews P, Tomasz A, Adamsson I, Aires de Sousa M, et al. Molecular typing of methicillin-resistant Staphylococcus aureus by pulsed-field gel electrophoresis: comparison of results obtained in a multilaboratory effort using identical protocols and MRSA strains. Microb Drug Resist. 2000;6:189-98.

25. Tenover FC, Arbeit RD, Goering RV, Mickelsen PA, Murray BE, Persing DH, et al. Interpreting chromosomal DNA restriction patterns produced by pulsedfield gel electrophoresis: criteria for bacterial strain typing. J Clin Microbiol. 1995:33:2233-9.

26. Enright MC, Day NP, Davies CE, Peacock SJ, Spratt BG. Multilocus sequence typing for characterization of methicillin-resistant and methicillin-susceptible clones of Staphylococcus aureus. J Clin Microbiol. 2000;38:1008-15.

27. Milheiriço C, Oliveira DC, de Lencastre H. Update to the multiplex PCR strategy for assignment of mec element types in Staphylococcus aureus. Antimicrob Agents Chemother. 2007;51:3374-7.

28. Shopsin B, Mathema B, Alcabes P, Said-Salim B, Lina G, Matsuka A, et al. Prevalence of agr specificity groups among Staphylococcus aureus strains colonizing children and their guardians. J Clin Microbiol. 2003;41:456-9.

29. Lina G, Piémont Y, Godail-Gamot F, Bes M, Peter MO, Gauduchon V, et al. Involvement of Panton-Valentine leukocidin-producing Staphylococcus aureus in primary skin infections and pneumonia. Clin Infect Dis. 1999;29:1128-32.

30. Su C-T, Hsueh P-R, Wu V-C, Wang C-Y, Hu F-C, Chen Y-M, et al. Maintenance haemodialysis and delayed administration of appropriate antibiotics increase 30-day mortality among patients with non-hospital-acquired meticillin-resistant Staphylococcus aureus bacteraemia. Int J Antimicrob Agents. 2010;35:511-2.

31. Del Rio A, Cervera C, Moreno A, Moreillon P, Miró JM. Patients at risk of complications of Staphylococcus aureus bloodstream infection. Clin Infect Dis. 2009:48 Suppl 4:S246-53. 
32. Peña C, Fernández-Sabe N, Domínguez MA, Pujol M, Martinez-Castelao A, Ayats J, et al. Staphylococcus aureus nasal carriage in patients on haemodialysis: role of cutaneous colonization. J Hosp Infect. 2004;58:20-7.

33. Kozioł-Montewka M, Szczepanik A, Baranowicz I, Jóźwiak L, Ksiazek A, Kaczor D. The investigation of Staphylococcus aureus and coagulase-negative staphylococci nasal carriage among patients undergoing haemodialysis. Microbiol Res. 2006;161:281-7.

34. Laupland KB, Ross T, Gregson DB. Staphylococcus aureus bloodstream infections: risk factors, outcomes, and the influence of methicillin resistance in Calgary, Canada, 2000-2006. J Infect Dis. 2008;198:336-43.

35. National Institutes of Health, National Institutes of Diabetes \& Digestive \& Kidney Disease D of KU\& HD. USRDS 2011 Annual Data Report: Atlas of Chronic Kidney Disease and End-Stage Renal Disease in the United States. 2011.

36. Ethier J, Mendelssohn DC, Elder SJ, Hasegawa T, Akizawa T, Akiba T, et al. Vascular access use and outcomes: an international perspective from the Dialysis Outcomes and Practice Patterns Study. Nephrol Dial Transplant. 2008;23:3219-26.

37. Lewis $T$, Chaudhry R, Nightingale $P$, Lambert $P$, Das I. Methicillin-resistant Staphylococcus aureus bacteremia: epidemiology, outcome, and laboratory characteristics in a tertiary referral center in the UK. Int J Infect Dis. 2011;15:e131-5.

38. Miskulin D. Characterizing comorbidity in dialysis patients: principles of measurement and applications in risk adjustment and patient care. Perit Dial Int. 2005;25:320-32.

39. Hsu DI, Hidayat LK, Quist R, Hindler J, Karlsson A, Yusof A, et al. Comparison of method-specific vancomycin minimum inhibitory concentration values and their predictability for treatment outcome of meticillin-resistant Staphylococcus aureus (MRSA) infections. Int J Antimicrob Agents. 2008;32:378-85.

40. Chen S-Y, Liao C-H, Wang J-L, Chiang W-C, Lai M-S, Chie W-C, et al. Method-specific performance of vancomycin MIC susceptibility tests in predicting mortality of patients with methicillin-resistant Staphylococcus aureus bacteraemia. J Antimicrob Chemother. 2014;69:211-8.

41. Kan L-P, Lin J-C, Chiu S-K, Yeh Y-C, Lin T-Y, Yang Y-S, et al. Methicillin-resistant Staphylococcus aureus bacteremia in hemodialysis and nondialysis patients. J Microbiol Immunol Infect 2014 Feb;47(1):15-22

42. Walraven CJ, North MS, Marr-Lyon L, Deming P, Sakoulas G, Mercier R-C. Site of infection rather than vancomycin MIC predicts vancomycin treatment failure in methicillin-resistant Staphylococcus aureus bacteraemia. J Antimicrob Chemother. 2011;66:2386-92.

43. Wiese L, Mejer $N$, Schønheyder HC, Westh $H$, Jensen AG, Larsen AR, et al. A nationwide study of comorbidity and risk of reinfection after Staphylococcus aureus bacteraemia. J Infect. 2013;67:199-205.

44. Lamagni TL, Potz N, Powell D, Pebody R, Wilson J, Duckworth G. Mortality in patients with meticillin-resistant Staphylococcus aureus bacteraemia, England 2004-2005. J Hosp Infect. 2011;77:16-20.

45. Gasch O, Camoez M, Dominguez MA, Padilla B, Pintado V, Almirante B, et al. Predictive factors for mortality in patients with methicillin-resistant Staphylococcus aureus bloodstream infection: impact on outcome of host, microorganism and therapy. Clin Microbiol Infect. 2013;19:1049-57.

46. Nielsen LH, Jensen-Fangel S, Benfield T, Skov R, Jespersen B, Larsen AR, et al. Risk and prognosis of Staphylococcus aureus bacteremia among individuals with and without end-stage renal disease: a Danish, population-based cohort study. BMC Infect Dis. 2015;15:6.

47. Ludwig F, Edwards B, Lawes T, Gould IM. Effects of storage on vancomycin and daptomycin MIC in susceptible blood isolates of methicillin-resistant Staphylococcus aureus. J Clin Microbiol. 2012;50:3383-7.

\section{Submit your next manuscript to BioMed Central and take full advantage of:}

- Convenient online submission

- Thorough peer review

- No space constraints or color figure charges

- Immediate publication on acceptance

- Inclusion in PubMed, CAS, Scopus and Google Scholar

- Research which is freely available for redistribution

Submit your manuscript at www.biomedcentral.com/submit 